\title{
Increasing penetration of renewable and distributed electricity generation and the need for different network regulation ${ }^{2 / 2}$
}

\author{
J. de Joode ${ }^{a, b, *}$, J.C. Jansen ${ }^{\text {a }}$, A.J. van der Welle ${ }^{\text {a }}$, M.J.J. Scheepers ${ }^{\text {a }}$ \\ ${ }^{a}$ Energy research Centre of the Netherlands (ECN), Amsterdam, the Netherlands \\ ${ }^{\mathrm{b}}$ Faculty of Technology, Policy and Management, Delft University of Technology, the Netherlands
}

\section{A R T I C L E I N F O}

\section{Article history:}

Received 24 December 2008

Accepted 9 March 2009

Available online 7 May 2009

Keywords:

Distributed generation (DG)

Distribution system operator (DSO)

Network regulation

\begin{abstract}
A B S T R A C T
The amount of decentralised electricity generation (DG) connected to distribution networks increases across EU member states. This increasing penetration of DG units poses potential costs and benefits for distribution system operators (DSOs). These DSOs are regulated since the business of electricity distribution is considered to be a natural monopoly. This paper identifies the impact of increasing DG penetration on the DSO business under varying parameters (network characteristics, DG technologies, network management type) and argues that current distribution network regulation needs to be improved in order for DSOs to continue to facilitate the integration of DG in the network. Several possible adaptations are analysed.
\end{abstract}

(c) 2009 Elsevier Ltd. All rights reserved.

\section{Introduction}

In European member states, the public goal of a sustainable electricity system is supported by several EU Directives, culminating in a number of technology-specific member state support schemes for renewable-based electricity generation (RES-E) and co-generation of electricity and heat (CHP). This drives the growth of distributed generation (DG) to significant levels. In this paper we follow Ackermann (2001) in defining DG: "Distributed generation is an electric power source connected directly to the distribution network or on the customer site of the meter". General support for this transition is justified when overall benefits for society (a more sustainable electricity system and higher level of network reliability and security of supply) exceed overall costs. Pepermans et al. (2005) provide an overview of the benefits and challenges brought about by an increasing amount of distributed electricity generation. The integration of DG in the electricity system poses benefits and costs to electricity market actors (DG operators, operators of the distribution and transmission networks, etc.). An important issue is the distribution of these benefits and costs over the different actors. An important part can consist of network integration costs due to an increasing number of DG connections. Different methods exist for the

\footnotetext{
An early version of this paper was presented at the 9th European IAEE conference in Florence, Italy in June 2007 (De Joode et al. 2007). The authors thank session participants at the 9th European IAEE conference in Florence for their comments.

* Corresponding author at: P.O. Box 56890, 1040 AW Amsterdam, the Netherlands. Tel.: +31 2245 68250; fax: +31 224568339

E-mail address: dejoode@ecn.nl (J. de Joode).
}

distribution of connection and network upgrading costs over DG operators and distribution system operators. These run from shallow charges-where DG operators only pay for direct connection costs - to deep charges-where DG operators bear the full cost of connection and network upgrades elsewhere in the distribution network. ${ }^{1}$ In this paper we assume a shallow charging approach, i.e. the (consumer or) DG operator connected to the network does not bear the full cost burden of connection. This assumption largely corresponds with the connection charging regime applied across EU member states (Skytte and Ropenus 2007). It is observed that the majority of the EU member states under study apply shallow or 'shallowish' connection charging on the distribution network level.

The role of the distribution system operator (DSO) in the transition to an electricity supply system increasingly based on distributed electricity generation is crucial. The DSO facilitates both connection of DG to, and the feed-in of electricity from DG in the distribution network. A large( $r$ ) cost burden for the DSO could therefore very well act as a barrier in continuing the integration of DG in electricity systems (Woodman and Baker 2008). A systematic qualitative and quantitative economic assessment of the impact-in terms of costs and benefits-of an increasing penetration of DG in the distribution network on the position of the DSO is, to our knowledge, still lacking in current literature. This paper contributes to filling this gap.

\footnotetext{
${ }^{1}$ For an overview of charging methodologies we refer to Knight et al. (2005). Barth et al. (2008) discuss the distribution of costs over market actors following connections of renewable electricity generating units in general (connected to transmission or distribution networks).
} 


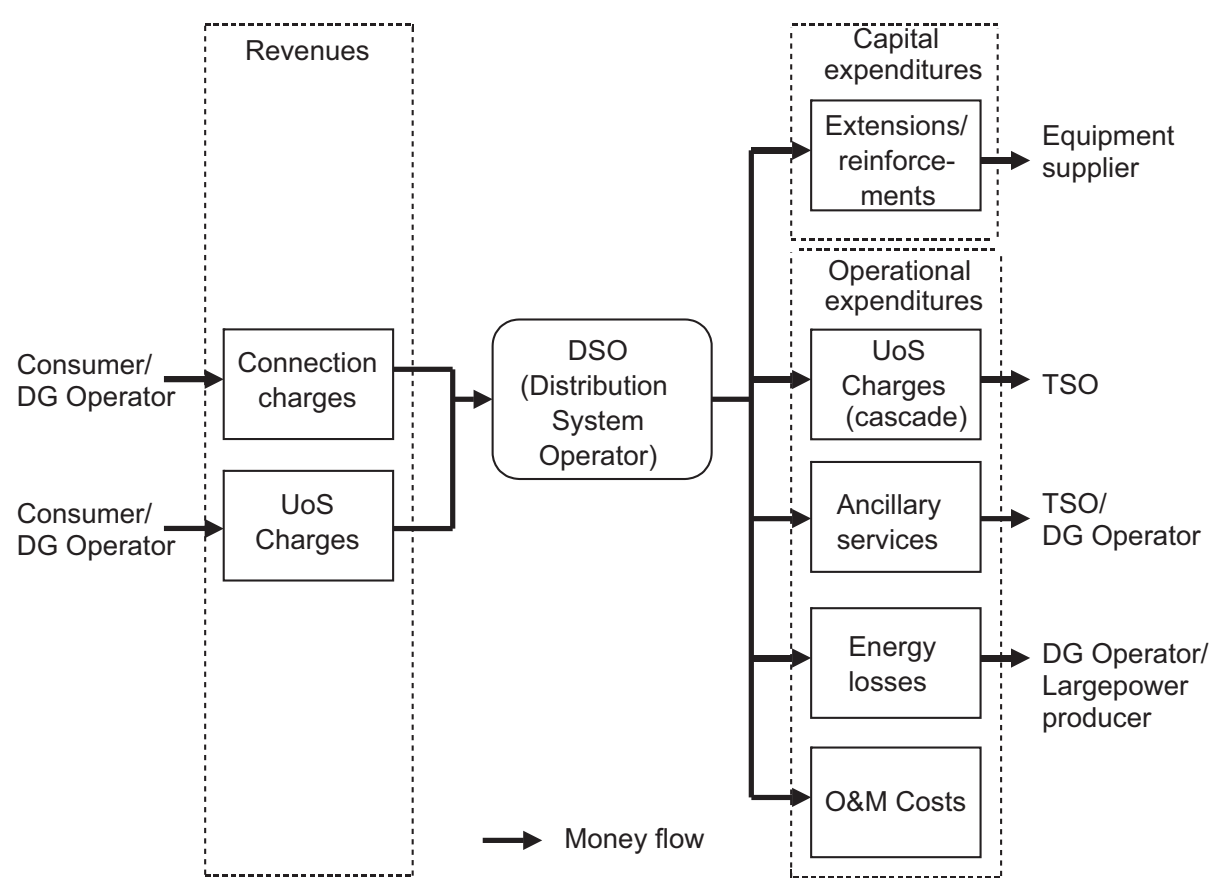

Fig. 1. Conceptual representation of the DSO business model

The goal of this paper is to get insight in the impact of an increasing penetration of DG in distribution networks on the operator of the network, the DSO. To this end we take a quantitative approach by using output data from a load flowbased generic distribution network model in a spread-sheet model representing the DSO business, i.e. the financial situation of the DSO. We first use this model to quantify the impact within the current regulatory regime and then analyse a number of adaptations to this regime to see whether adaptations can mitigate possible negative impacts on the DSO.

In general, a business model refers to the particular business strategy applied to recover expenditures, including a proper rate of return on investment, with revenues. Fig. 1 presents the conceptual representation of the business model of a DSO in our analysis.

The DSO is a regulated business since the distribution of electricity is considered to be a natural monopoly due to economic characteristics. The distribution of electricity is highly asset-specific involving a large share of capital expenditures relative to operational expenditures, and concerns long lifetime of investment. In other words: general textbook economics does not apply here. Prevailing distribution network regulation regimes across EU countries prevent DSOs from acting as a monopolist. Third party access rules ensure non-discriminatory access to all distribution network users whilst some form of revenue-cap or price-cap regulation prevents the DSO from charging access and use of system fees at 'monopolistic' levels. Hence, revenues are subject to regulatory review.

DSO expenditures are divided into two categories: (a) capital expenditures, and (b) operational expenditures. Capital expenditures include investments in distribution network assets such as transformers, switchboards, and cables, and the consequential depreciation costs and remuneration of debt. Operational expenditures encompass costs due to use of the transmission network, distribution losses, costs of ancillary services, and operational and maintenance costs of assets. ${ }^{2}$

\footnotetext{
${ }^{2}$ Commercial costs related to energy measurement and billing to final consumers are also considered to be operational expenditures. Obviously, also
}

Apart from the technical exposition on the, largely by regulation determined, revenues and expenditures of a DSO, the general DSO philosophy of network management is of importance in analysing the impact of increasing penetration of DG in distribution networks. We distinguish between (i) passive network management and (ii) active network management.

The current network management approach is based on an equal treatment of both consumers and distributed generators that want to be connected to the distribution network. New DG connections influence electricity flows in the distribution network. The network needs to be dimensioned on peak generating capacity of the DG unit resulting, in some cases, in incremental reinforcement in the network by the DSO. This is also called the passive network management approach, or 'fit and forget' approach: when DG enters the distribution network, upgrade investments based on peak DG output are undertaken and for the remainder any electricity production from the unit is taken as given.

An alternative approach is provided with an active network management philosophy (McDonald 2008). This approach is based on the concept of intelligent networks where technological innovations on power equipment and ICT are combined to allow for a more efficient use of distribution network capacity. In addition, it is characterised by active involvement of both consumers and distributed generators: load and generation characteristics are taken into account in network operations and planning. When confronted with new connections the active DSO explicitly recognizes the network contribution of electricity consuming and producing entities in its network planning and includes this in investment decision-making.

This paper proceeds as follows. In Section 2 we present the applied methodology used in the analysis. Section 3 contains our analysis of the issue and presents quantitative results. Section 4 concludes and provides some policy recommendations.

(footnote continued)

these costs are impacted by DG penetration since DG operators are 'network customers' as well. However, an analysis on the impact of DG penetration on these commercial costs is beyond our scope. 


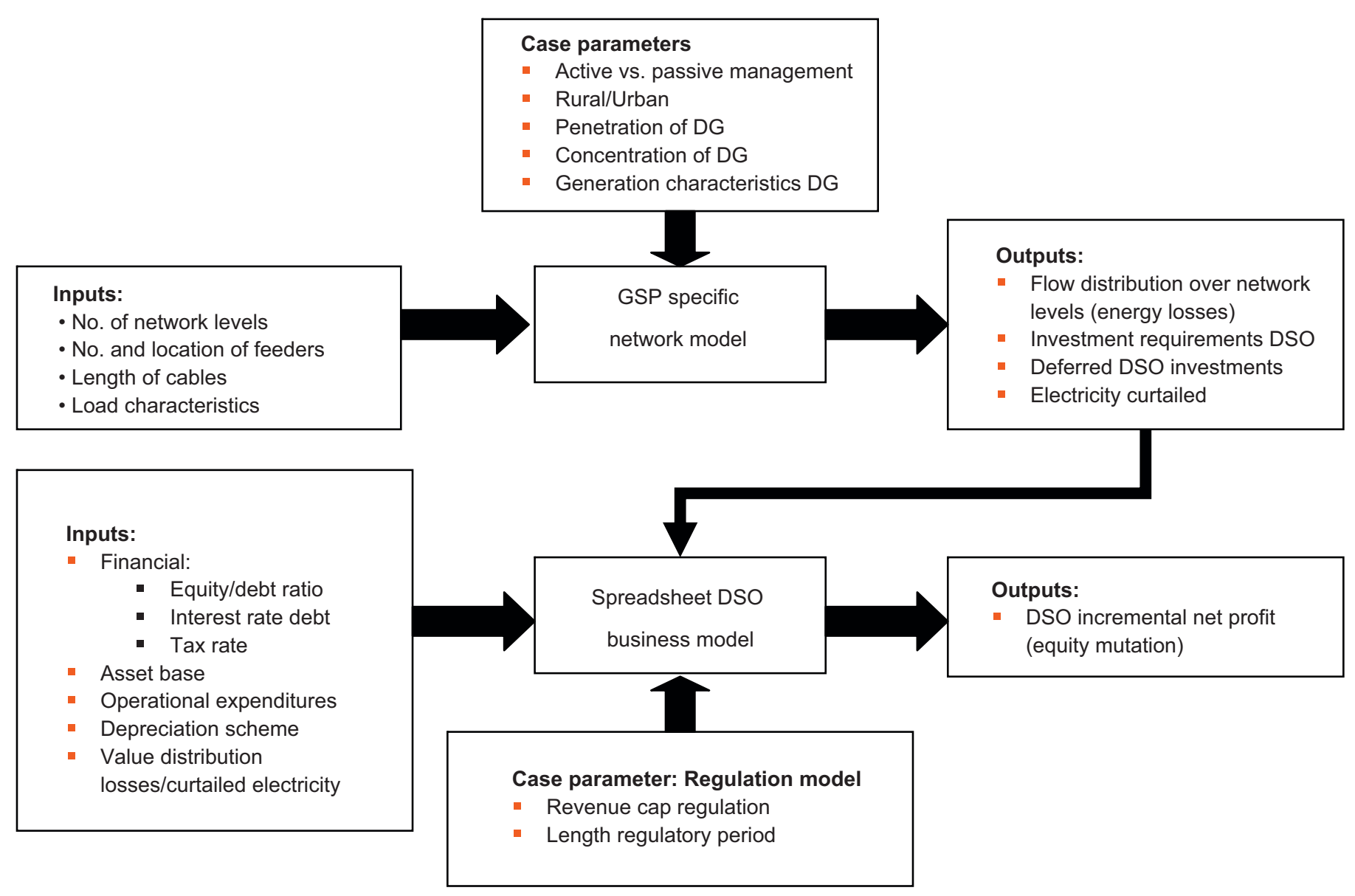

Fig. 2. Schematic overview of the linkages between the generic network model and the spreadsheet model

\section{Methodology}

\subsection{Introduction}

In order to evaluate the impact of an increasing penetration of distributed electricity generators on the DSO we deploy a combination of two different models; a load flow-based generic distribution network model representing a distribution network and a DSO spreadsheet model representing the financial position of the DSO.

Fig. 2 shows the basic input-output relation between the generic distribution network model and the DSO spreadsheet model.

\subsection{Generic distribution network model}

The generic distribution network model is based on the topology and design of an 'average' UK distribution network ${ }^{3}$ that is connected to a 'grid supply point' (GSP). ${ }^{4}$ The distribution network consists of various cascading low voltage level networks with substations and transformers between each of these networks. The voltage networks are modelled as radial networks

\footnotetext{
${ }^{3}$ The model was used to analyse the impact of DG penetration in two specific EU countries: Finland and the UK (Cao et al. 2006). A comparison of results of the case studies provides insight into the robustness of results. Since the UK network better resembles other European countries' distribution network topology and design, the UK results have been used in our analysis. However, a similar analysis as performed in this report can be undertaken for other countries as well.

${ }^{4}$ A GSP is a point of connection between the distribution network and the higher voltage transmission network.
}

from the GSP to the end-users. A graphical representation of a generic distribution network, with various loads and distributed generators connected, is shown in Fig. 3. In our analysis, the distribution network is dimensioned at a total load of 1155 GWh per year (being equal to the load of one grid supply point). Here we do not discuss the model further in depth. For an extensive model and data input description we refer to Pudjianto et al. (2006).

\subsection{DSO spreadsheet model}

The DSO spreadsheet model represents the financial statements of a DSO. It lists expenditures and costs and calculates net profit over a longer period of time. The model has the following basic characteristics.

First, the model is incremental in the sense that it does not explicate the costs and revenues related to 'business as usual' but only the incremental costs and revenues caused by the increase of DG in the distribution network. This enables a strict assessment of the impact of DG on the DSO. Second, the model is dynamic: it captures a number of regulatory periods with each covering a number of years. This approach is in line with current practice where the DSO, being a regulated actor, is confronted with a network performance assessment by the regulatory authority once per regulatory period. Third, the specific regime of distribution network regulation assumed to apply for the studied DSO is based on incentive based regulation and more in particular, based on a revenue-cap system. For a discussion on the types of regulatory regimes we refer to Jansen et al. (2007). Our choice for this specific regime can be justified by an EU wide observed 


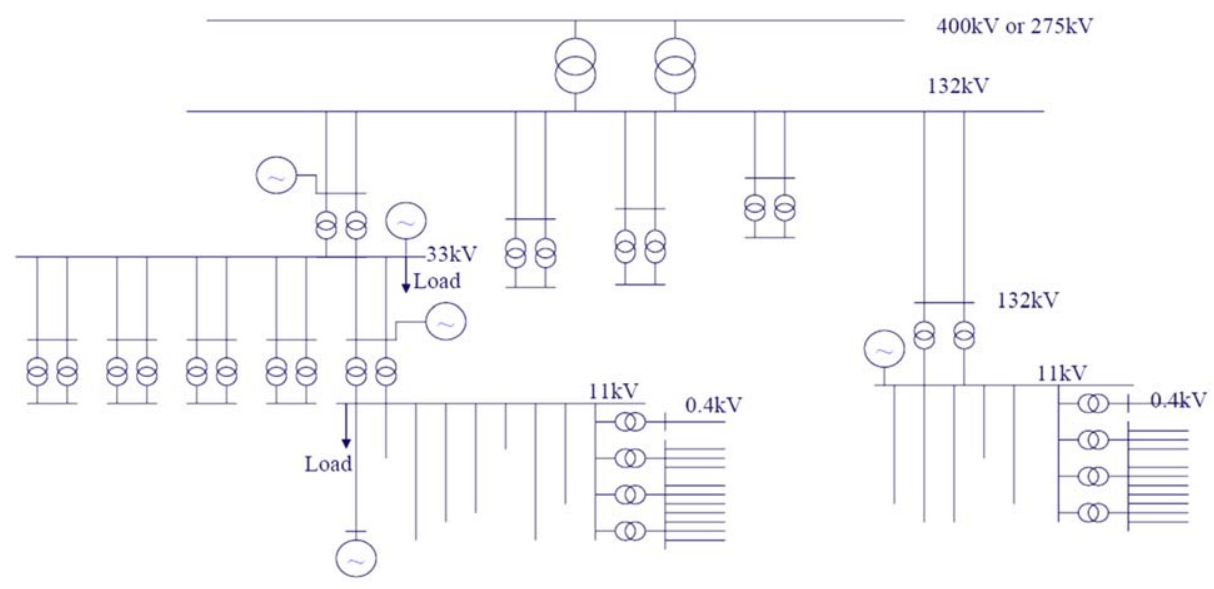

Fig. 3. Representation of the module-based distribution network design in the generic distribution network model (Pudjianto et al. 2006).

tendency to move from traditional cost-plus regimes to incentive based regimes (Skytte and Ropenus 2007). Below we go into more detail on this type of distribution network regulation since this is vital for the remainder of the paper.

Incentive based regulation trough a revenue cap basically implies the following: the DSO is only allowed to receive a maximum total allowed revenue (TAR) in return for its services in one year, with the TAR in one year being equal to the TAR in the previous period corrected for (i) a requirement on improved efficiency performance, (ii) change in overall price level (inflation), and (iii) optional compensation schemes for adverse developments in demand. ${ }^{5}$ Since the DSO model uses and presents nominal prices the revenue cap scheme included in the DSO model does not contain a correction for inflation. The foregoing results in the following formula:

$T A R_{t}=T A R_{t-1}(1-X) \pm A F$

Where $T A R_{t}$ is the Total allowed revenue in year $t, X$ the Required yearly improvement in efficiency performance, $A F$ the Adjustment factor

The total allowed revenue in the starting year is dependent on the total regulated asset base (RAB), the weighted average cost of capital (WACC) and the operational expenditures. The RAB represents the value of the DSOs asset base.

$T A R_{0}=f(R A B, W A C C, O P E X)$

In traditional incentive-based regulation DSOs are not allowed to include all new investments into the regulated asset base: i.e. no automatic pass-through of investment costs to end-consumers is allowed for. However, some EU member states do allow for 'exceptional' investments to be included in the RAB. ${ }^{6}$ The only country to have such a rule regarding specifically DG related investment is the UK. In the remaining analysis we assume that DSOs are not allowed to pass-through the costs of DG related incremental network reinforcement costs.

\footnotetext{
${ }^{5}$ In the DSO business model, it is assumed that no adjustment factor, for example related to demand growth, is currently used. Further on in this report we analyse the use of an adjustment factor to compensate DSOs for the possible negative impact of DG penetration in the distribution network.

${ }^{6}$ This is also referred to as incentive-based regulation based on the 'so-called' building blocks' approach. For a more elaborate discussion on this specific approach we refer to Jansen et al. (2007).
}

\subsection{Parameterisation of cases}

In order to explore the impact of DG penetration on the DSO business we identify different case parameters that simulate different conditions under which DSOs are faced with increasing DG penetration. We distinguish between different levels of DG in the network, different concentration ratios for DG units, different DG generation profiles, different network topologies and different types of network management. Below, we briefly comment on each case parameter.

Firstly, the level of $D G$ penetration in the network indicates the amount of peak DG capacity in the network. This amount ranges from 50 to 100 to $200 \mathrm{MW}$. Given the total level of demand of 1155 GWh in the distribution network, and assumed load factors for intermittent (30\%) and non-intermittent (60\%) distributed electricity generation. This results in a range of penetration rate of 10 to $90 \%$ of total load.

Secondly, the concentration of DG capacity indicates whether the spatial concentration of DG within the distribution network is either high or low, where concentration refers to the physical location of the DG unit. ${ }^{7}$

Thirdly, the share of intermittent generation in total DG capacity is considered an important case parameter. Distributed electricity producing units vary in electricity generation profile. Whereas some DG units can produce at relatively constant rate, others are volatile in electricity generation. The former category of units is referred to as being non-intermittent DG; the latter is referred to as intermittent DG. A related distinction is that between controllable and non-controllable DG units in the sense that DG units producing electricity intermittently can in principle be controllable. For example, small-scale CHP units at industrial sites are in principal controllable (and hence can respond to changes in electricity prices) but since their electricity production follows industrial heat demand, the resulting electricity production profile is referred to as being intermittent. Within the generic distribution network model, all DG connected to the distribution network is either fully non-intermittent or fully intermittent and there is no distinction between controllable and non-controllable, which is a simplification of reality where an indefinite portfolio of DG units with different electricity generating profiles exist. Hence,

\footnotetext{
${ }^{7}$ The two levels of concentration relate to the 'density' levels distinguished in Cao et al. (2007). They distinguish four levels varying from 'low' to 'medium-low', 'medium-high' and 'high'.
} 
in our analysis we explore the two extremes of $0 \%$ intermittency of total DG capacity and 100\% intermittency of total DG capacity.

Fourthly, we consider the type of network to be of influence when assessing the impact of DG penetration on the distribution network. A rural network typically consists of longer lines and less concentrated loads than urban networks. Jenkins et al. (2000) mention two different technical barriers for increasing levels of DG. In rural areas voltage management and thermal rating issues can hinder further DG deployment, whether urban areas experience system fault level issues. This implies different requirements for DSO investments in both networks. ${ }^{8}$

Fifthly and lastly, as has been argued earlier in the paper, the type of network management approach, passive or active bears impact on the way in which DG is integrated in the distribution system. Passive network management can be described as a 'fit and forget' network management approach where network requirements caused by new DG connections are met by 'simple' capacity expansion. Active network management on the other hand uses 'intelligence' in the network to cope with new DG connections.

\section{Analysis and results}

\subsection{Introduction}

We have analyzed the impact of DG penetration on the financial position of the DSO for a number of cases. Table 1 gives an overview of the studied cases and the taken values for the earlier described case parameters. The different cases can be interpreted as different type of distribution networks/distribution system operators.

The general analysis can be divided into two distinct steps. In the first step, the generic distribution network model calculates the physical impact of DG penetration: i.e. the impact on distribution losses, on required or deferred capacity upgrades etc. In the second step, these results are translated by the spreadsheet model in a net financial impact on the DSO given a regulatory regime based on incentive regulation through a revenue-cap. The results of the first step are analysed and discussed in Cao et al. (2007). Here we focus on the results of the second step of analysis.

\subsection{Results of the impact analysis}

The results of the analysis are depicted in Table 2 .

The impact of DG on the (regulated) financial position of the DSO is indicated as a percentage change in the net profit of the DSO in a situation where no DG enters the distribution network. Recall that costs and revenues resulting from 'normal' business, i.e. a distribution network where no DG is present in the network, are not analyzed: the spreadsheet model is incremental in nature. For example, for case \#1 it is reported that an integration of 100 MW of concentrated and intermittent DG capacity in a rural distribution network operated under a passive network management philosophy, results in a $7.8 \%$ increase in net profit for the DSO compared to the no DG penetration case. The table reports two results: one including and one excluding the potential replacement value of integrated DG. The replacement value is created when DG penetration at one point in time, prevents or defers the (replacement) investment in network capacity upgrades at a later

\footnotetext{
${ }^{8}$ For specific technical description of the problems DG penetration can pose for network operators and the consequences for investment requirements we refer to Cao et al. (2007).
}

Table 1

List of case parameters and values.

\begin{tabular}{lll}
\hline Case parameter & Values & Unit \\
\hline 1. Level of DG & {$[50,100,200]$} & {$[\mathrm{MW}]$} \\
2. Concentration of DG & {$[$ low, high] } & {$[$ Discrete] } \\
3. Share of intermittent generation & {$[0,100]$} & {$[\%]$} \\
4. Type of network & {$[$ rural, urban] } & [Discrete] \\
5. Type of network management & [passive, active] & [Discrete] \\
\hline
\end{tabular}

point in time (Mendez et al., 2006). For example, continuous load growth due to increasing electricity demand would in due time require new investments in the substation that connects the distribution with the transmission network, unless increasing DG levels within the network could cover the additional load growth. The lifetime of the electrical equipment in the distribution network influences the potential replacement value in the same manner. Hence, due to the uncertain development of load growth in the distribution network and the technical state of electrical equipment we choose to present a result including $0 \%$ and $100 \%$ of the potential replacement value. In reality, the actual value will be somewhere in-between.

Results show that, if the potential value of deferred investments is not taken into account, DSOs operating under a passive network management regime generally do not profit from the presence of DG in their distribution network. Although low DG penetration levels do benefit the DSO somewhat, higher penetration levels result in a negative overall impact. The concentration of DG within the network is a particular influential factor: the more concentrated the presence of DG in the distribution network, the more negative the impact. The driver for the generally positive results for low penetration levels and the generally negative results for high penetration levels are distribution losses.

DSOs operating under an active network management philosophy are generally confronted with comparable results as the passive network management case. Penetration of DG in the network is favourable for the DSO for low penetration levels, but becomes unfavourable the higher the penetration rate and the more concentrated the DG in the network. However, it should be noted that the negative results are relatively small for the majority of the cases analyzed: the net impact of DG penetration is mostly within the range of $8 \%$ of the 'business as usual' profit DSOs make.

The added value of DG with respect to the investment deferral for connections to the higher voltage network levels can be substantial. However, the realization of this positive value for the DSOs is dependent on a larger number of non-DG related factors and is beyond the scope of this investigation (e.g. load growth dynamics and the status of interconnection equipment). However, considering the maximum replacement values of DG, it can be expected that the overall impact of DG penetration on the DSO business can be neutral or positive in the majority of cases. Observing the differential impact on the DSO under passive and active network management we conclude that there is an implicit incentive for the DSO to adopt an active network management approach in a number of cases, in particular the case where DG penetration is low or medium.

The observation that an increased penetration of DG is indeed negative for the DSO does not automatically justify adaptation of distribution network regulation. When assessing the necessity of such adaptation one also needs to take into account potential positive impacts on the DSO brought about by increased DG penetration. These could for example emerge in the field of ancillary services provision and demand response mechanisms (Strbac 2008, Jansen et al. 2007). The DSO can for example act as an intermediary in the provision of these types of services due to 
Table 2

Impact of DG deployment on the DSO's revenue relative to 'business as usual'.

\begin{tabular}{|c|c|c|c|c|c|c|c|}
\hline \multirow[t]{2}{*}{ Case } & \multicolumn{5}{|l|}{ Parameter } & \multicolumn{2}{|l|}{ Impact on DSO net profit } \\
\hline & $\begin{array}{l}\text { Level DG } \\
(\mathrm{MW})\end{array}$ & $\begin{array}{l}\text { Net-work } \\
\text { type }\end{array}$ & $\begin{array}{l}\text { Concentration } \\
\text { DG }\end{array}$ & Type of DG & $\begin{array}{l}\text { Management } \\
\text { type }\end{array}$ & $\begin{array}{l}\text { Excluding potential } \\
\text { deferred investment (\%) }\end{array}$ & $\begin{array}{l}\text { Including potential } \\
\text { deferred investment (\%) }\end{array}$ \\
\hline 1 & 100 & Rural & High & Intermittent & Passive & 7.8 & 29.6 \\
\hline 2 & 100 & Rural & High & Intermittent & Active & 9.2 & 31.0 \\
\hline 3 & 100 & Rural & High & Non-intermittent & Passive & -7.0 & 14.8 \\
\hline 4 & 100 & Rural & High & Non-intermittent & Active & -6.7 & 15.1 \\
\hline 5 & 50 & Rural & Low & Intermittent & Passive & 2.1 & 12.9 \\
\hline 6 & 50 & Rural & Low & Intermittent & Active & 2.1 & 12.9 \\
\hline 7 & 50 & Rural & Low & Non-intermittent & Passive & 5.2 & 15.9 \\
\hline 8 & 50 & Rural & Low & Non-intermittent & Active & 5.2 & 15.9 \\
\hline 9 & 200 & Rural & High & Intermittent & Passive & -16.2 & 1.0 \\
\hline 10 & 200 & Rural & High & Intermittent & Active & -21.6 & -4.4 \\
\hline 11 & 200 & Rural & High & Non-intermittent & Passive & -44.7 & -27.5 \\
\hline 12 & 200 & Rural & High & Non-intermittent & Active & -57.3 & -40.0 \\
\hline 13 & 100 & Rural & Low & Intermittent & Passive & -4.3 & 17.6 \\
\hline 14 & 100 & Rural & Low & Intermittent & Active & -4.5 & 17.4 \\
\hline 15 & 100 & Rural & Low & Non-intermittent & Passive & 0.3 & 22.2 \\
\hline 16 & 100 & Rural & Low & Non-intermittent & Active & 0.3 & 22.2 \\
\hline 17 & 100 & Urban & High & Intermittent & Passive & -1.2 & 20.6 \\
\hline 18 & 100 & Urban & High & Intermittent & Active & 4.6 & 26.4 \\
\hline 19 & 100 & Urban & High & Non-intermittent & Passive & -10.5 & 11.3 \\
\hline 20 & 100 & Urban & High & Non-intermittent & Active & -3.8 & 18.0 \\
\hline 21 & 50 & Urban & Low & Intermittent & Passive & -8.4 & 2.3 \\
\hline 22 & 50 & Urban & Low & Intermittent & Active & -1.6 & 9.1 \\
\hline 23 & 50 & Urban & Low & Non-intermittent & Passive & 2.6 & 13.4 \\
\hline 24 & 50 & Urban & Low & Non-intermittent & Active & 0.4 & 11.2 \\
\hline 25 & 200 & Urban & High & Intermittent & Passive & -26.4 & -9.2 \\
\hline 26 & 200 & Urban & High & Intermittent & Active & -32.9 & -15.7 \\
\hline 27 & 200 & Urban & High & Non-intermittent & Passive & -41.1 & -23.9 \\
\hline 28 & 200 & Urban & High & Non-intermittent & Active & -51.9 & -34.6 \\
\hline 29 & 100 & Urban & Low & Intermittent & Passive & -10.6 & 11.3 \\
\hline 30 & 100 & Urban & Low & Intermittent & Active & -2.3 & 19.6 \\
\hline 31 & 100 & Urban & Low & Non-intermittent & Passive & 1.2 & 23.2 \\
\hline 32 & 100 & Urban & Low & Non-intermittent & Active & 0.1 & 22.1 \\
\hline
\end{tabular}

the network information acquired through implementation of active network management. These services would imply a new source of revenues for the DSO that might compensate for negative impact with regard to network operations and investment due to increased DG penetration..$^{9}$ Additional research on the significance of this type of business opportunities for DSOs is required. For now we assume that these opportunities do not (fully) compensate the negative impact of the type shown above and therefore proceed to propose possible improvements for current network regulation that can mitigate the negative impact.

\subsection{Results of analysis on regulatory improvements}

The negative impact of DG integration on the DSO's financial position as reported above may hamper the deployment of DG resulting in a 'conflict' with the national and European policy objectives for CHP and RES-E. To solve this problem the additional costs of DG integration should be socialized among all customers connected to the network, i.e. electricity consumers and generators alike. The network costs for connecting and integrating DG is then treated in the same way as network costs related to electricity consumption. This reflects the role of the distribution network: providing access to the electricity market for consumers and (distributed) generators under similar conditions. The extra network costs induced by DG connections can be allocated to consumers and DG operators through the use of system

\footnotetext{
${ }^{9}$ Weber and Vogel (2008) assess the benefits of system services provision for
} certain DG technologies. charges. These tariffs (connection charges and use of system charges) are calculated from the TAR by taking into account the number of connections, size of connections, amounts of kWh and $\mathrm{kW}_{\text {peak }}$, etc.

With the revenue cap formula (Equation 1) as a starting point, five different ways to compensate for DG penetration have been identified (De Joode et al. 2007). The DSO spreadsheet model was applied to test the effectiveness of four particular improvement options (see below). ${ }^{10}$ Table 2 shows the DSO's revenue in case of DG penetration relative to 'business as usual' for the four regulatory improvement options in comparison to a reference case excluding the potential deferred investment value. The four options shown in Table 2 are:

1. An allowance in the regulated asset base (RAB) for the DSO for DG related investments. This option compensates for the negative impact of DG penetration on capital investment but not on operational expenditures. A pass-through of DG related investments less than $100 \%$ is used so that an economic incentive remains to limit these investments. A 30\% passthrough is used for a low, 70\% for a medium and 90\% for a high DG penetration rate. This type of compensation measure may be described by the following formula ${ }^{11}$ :

$$
T A R_{t}=T A R_{t-1}(1-X)+y \% \cdot I_{t}^{D G}
$$

\footnotetext{
${ }^{10}$ A fifth theoretical option is to consider DG as a cost driver in the DSO benchmarking exercise. However, the model is not capable of analysing this option.

${ }^{11}$ Assuming that demand growth is zero so that the adjustment factor $\mathrm{AF}$ (see equation 1 ) is equal to zero.
} 
Table 3

The DSO's revenue relative to 'business as usual' in the reference case (without potential deferred investment value) and four regulatory improvement options.

\begin{tabular}{|c|c|c|c|c|c|c|c|c|c|c|}
\hline \multirow[t]{2}{*}{ Case } & \multicolumn{5}{|l|}{ Parameter } & \multirow{2}{*}{$\begin{array}{l}\text { Reference } \\
\text { Impact on } \\
\text { DSO revenue } \\
(\%)\end{array}$} & \multicolumn{4}{|c|}{ Improvement options } \\
\hline & $\begin{array}{l}\text { Level DG } \\
(\mathrm{MW})\end{array}$ & $\begin{array}{l}\text { Net-work } \\
\text { type }\end{array}$ & $\begin{array}{l}\text { Concentration } \\
\text { DG }\end{array}$ & Type of DG & $\begin{array}{l}\text { Management } \\
\text { type }\end{array}$ & & $\begin{array}{l}\text { Allowance in } \\
\mathrm{RAB}(\%)\end{array}$ & $\begin{array}{l}\text { Quality } \\
\text { indicator (\%) }\end{array}$ & $\begin{array}{l}\text { Direct revenue } \\
\text { driver }(\%)\end{array}$ & $\begin{array}{l}\mathrm{RAB} \text { and direct } \\
\text { revenue driver (\%) }\end{array}$ \\
\hline 1 & 100 & Rural & High & Int. & Pas. & 7.8 & 10.6 & 12.7 & 9.9 & 17.2 \\
\hline 2 & 100 & Rural & High & Int. & Act. & 9.2 & 10.6 & 14.1 & 11.3 & 16.3 \\
\hline 3 & 100 & Rural & High & Int. & Act. & -7.0 & 1.0 & 11.5 & 9.3 & 9.1 \\
\hline 4 & 100 & Rural & High & Int. & Act. & -6.7 & -1.8 & 11.7 & 9.5 & 7.3 \\
\hline 5 & 100 & Rural & High & Int. & Act. & 2.1 & 2.1 & 4.6 & 3.2 & 4.5 \\
\hline 6 & 100 & Rural & High & Int. & Act. & 2.1 & 2.1 & 4.6 & 3.2 & 4.5 \\
\hline 7 & 100 & Rural & High & Int. & Act. & 5.2 & 5.2 & 10.1 & 6.2 & 9.8 \\
\hline 8 & 100 & Rural & High & Int. & Act. & 5.2 & 5.2 & 10.1 & 6.2 & 9.8 \\
\hline 9 & 100 & Rural & High & Int. & Act. & -16.2 & 2.8 & 4.7 & 5.4 & 8.9 \\
\hline 10 & 100 & Rural & High & Int. & Act. & -21.6 & -6.6 & -0.2 & 1.6 & 1.7 \\
\hline 11 & 200 & Rural & High & Non-int. & Pas. & -44.7 & -18.3 & -1.8 & 3.0 & -3.8 \\
\hline 12 & 200 & Rural & High & Non-int. & Act. & -57.3 & -36.4 & -13.6 & -8.2 & -19.0 \\
\hline 13 & 100 & Rural & Low & Int. & Pas. & -4.3 & -2.4 & 1.9 & -1.3 & 4.1 \\
\hline 14 & 100 & Rural & Low & Int. & Act. & -4.5 & -3.2 & 1.7 & -1.5 & 3.1 \\
\hline 15 & 100 & Rural & Low & Non-int. & Pas. & 0.3 & 3.7 & 16.7 & 14.5 & 12.0 \\
\hline 16 & 100 & Rural & Low & Non-int. & Act. & 0.3 & 2.5 & 16.6 & 14.5 & 11.2 \\
\hline 17 & 100 & Urban & High & Int. & Pas. & -1.2 & 3.8 & 4.1 & 1.3 & 11.7 \\
\hline 18 & 100 & Urban & High & Int. & Act. & 4.6 & 5.6 & 9.5 & 6.7 & 10.8 \\
\hline 19 & 100 & Urban & High & Non-int. & Pas. & -10.5 & 2.7 & 9.0 & 6.8 & 9.8 \\
\hline 20 & 100 & Urban & High & Non-int. & Act. & -3.8 & -1.0 & 13.7 & 11.5 & 8.2 \\
\hline 21 & 50 & Urban & Low & Int. & Pas. & -8.4 & -2.3 & -5.0 & -6.9 & 3.9 \\
\hline 22 & 50 & Urban & Low & Int. & Act. & -1.6 & 0.6 & 1.3 & -0.1 & 4.3 \\
\hline 23 & 50 & Urban & Low & Non-int. & Pas. & 2.6 & 2.6 & 5.3 & 1.5 & 7.3 \\
\hline 24 & 50 & Urban & Low & Non-int. & Act. & 0.4 & 2.3 & 5.3 & 1.5 & 8.2 \\
\hline 25 & 200 & Urban & High & Int. & Pas. & -26.4 & 7.2 & -4.7 & -2.5 & 9.7 \\
\hline 26 & 200 & Urban & High & Int. & Act. & -32.9 & 4.2 & -10.9 & -9.0 & 6.2 \\
\hline 27 & 200 & Urban & High & Non-int. & Pas. & -41.1 & 3.4 & 1.6 & 5.5 & 8.6 \\
\hline 28 & 200 & Urban & High & Non-int. & Act. & -51.9 & -2.8 & -8.4 & -2.8 & 1.6 \\
\hline 29 & 100 & Urban & Low & Int. & Pas. & -10.6 & -4.4 & -4.0 & -7.6 & 4.8 \\
\hline 30 & 100 & Urban & Low & Int. & Act. & -2.3 & -1.7 & 3.3 & 0.5 & 3.8 \\
\hline 31 & 100 & Urban & Low & Non-int. & Pas. & 1.2 & 1.2 & 17.6 & 15.4 & 10.6 \\
\hline 32 & 100 & Urban & Low & Non-int. & Act. & 0.1 & 1.2 & 16.5 & 14.3 & 10.2 \\
\hline
\end{tabular}

where

$y=$ Share of eligible DG related investments in distribution network assets

$I_{t}^{D G}=$ Total eligible DG related investments in distribution network assets in year $t$

2. Including an additional quality indicator through which DSOs are compensated for higher DG presence in their distribution network:

$T A R_{t}=T A R_{t-1}\left(1-X+K_{\text {Ind }}\right)$

The chosen value for the $K_{\text {Ind }}$ is $0.75 \%$ for a DG penetration level of $11 \%$ and $1.5 \%$ for $23 \%, 5 \%$ for $46 \%$ and $10 \%$ for $91 \%$ respectively.

Allowing one or more DG based direct revenue driver(s) in the revenue cap formula:

$T A R_{t}=T A R_{t-1}(1-X)+F_{1} \cdot k W^{D G}+F_{2} \cdot M W h^{D G}$

3. The allowance is based on the DG capacity $\left(F_{1}=2.5 € / \mathrm{kW}\right.$ for a low, $F_{1}=2 € / \mathrm{kW}$ for a medium and $F_{1}=1 € / \mathrm{kW}$ for high a DG penetration) and the electricity supply of DG $\left(F_{2}=0 € / M W h\right.$ for a low, $F_{2}=2.5 € / M W h$ for a medium and $F_{2}=3.5 € / M W h$ for a high DG penetration).

4. A combination of a special $R A B$ allowance and direct revenue driver. While the direct revenue driver in this scheme is still based on energy, the capacity based direct revenue driver is replaced by a special $R A B$ allowance for DG related investments:

$T A R_{t}=T A R_{t-1}(1-X)+y \% \cdot I_{t}^{D G}+F \cdot M W h^{D G}$
The rate for total eligible DG related investments $\left(\mathrm{I}_{\mathrm{t}}^{\mathrm{DG}}\right)$ is $50 \%$ and the direct revenue driver $(F)$ has the value of $2 € / \mathrm{MWh}$.

As the results of the analysis of improvement options shown in Table 3 indicate there is no 'one size fits all' solution for neutralizing the negative impact of DG penetration on DSO's revenue. Since the negative impact of through either operational expenditures (distribution losses) or capital expenditures (network upgrades) is dominant, a specific regulatory arrangement with compensatory elements based on either 'DG energy produced' or 'DG capacity connected' can not fully compensate the one DSO without unnecessarily 'overcompensating' other DSOs. The most successful regulatory improvement seems to be the combination of a special allowance and a direct revenue driver. When applying this option DSOs will be able to recover their costs. It should be noted that a minor 'overcompensation' of DSOs for the negative impact they experience from DG penetration in the network might effectively work as an incentive to optimally facilitate additional DG connections within their network.

\section{Conclusions and recommendations}

Our analysis has shown that increasing DG presence in distribution networks can (1) be either favourable or not, depending on certain parameters, and in addition, (2) creates new market opportunities that can ultimately lead to different DSO business models than currently observed. 
Firstly, we analyzed the economic impact of DG penetration in distribution networks on the DSO taking into account a number of network and DG characteristics. In addition, we included two different network management philosophies (passive and active network management) in the analysis.

(1) We find that DSOs operating under a passive network management regime generally do not profit from the presence of DG in their distribution network, except for low DG penetration levels: network reinforcement costs increase with the level of DG penetration.

(2) DSOs adopting active network management are generally faced with similar general results as DSOs operating adopting passive network management. Penetration of DG in the network is favourable for the DSO for low penetration levels, but becomes unfavourable the higher the penetration rate and the more concentrated the DG is in the distribution network.

(3) The added value of DG with respect to the deferral of investments at the grid supply point (the connection with the higher voltage level network) can be substantial. However, the realization of this positive value for the DSOs is dependent on a larger number of factors that are beyond the scope of our research. These relate to the load growth dynamics and the status of interconnection equipment (economic lifetime and depreciation so far). Considering the maximum replacement values of DG, it can be expected that the overall impact of DG penetration on the DSO business, can be neutral or positive in the majority of cases.

(4) Observing a differential impact on the DSO under passive and active network management we conclude that there is an implicit incentive for the DSO to adopt an active network management approach in a number of cases, in particular the case where DG penetration is low or medium. For cases with high DG penetration conclusions still cannot be drawn; more research is needed on the costs for the DSO when combining passive and active network management elements.

(5) Regarding the implications of the analysis on alternative regulatory arrangements we note that there are several cases where the DSO is negatively impacted by DG penetration, regardless of the network management philosophy. This implies that an alternative regulatory arrangement compensating the DSO for the negative impact might be warranted. Moreover, we have seen that in such cases the incentive for DSOs to move from a passive network management approach to an active network management approach is not always present.

(6) From the analysis on regulatory improvements we found that an alternative regulatory arrangement based on a combination of a special $\mathrm{RAB}$ allowance and a direct revenue driver seems most successful in compensating the negative DG impact on DSOs. When applying this regulatory improvement DSOs will be able to recover their costs and at the same time are stimulated to connect existent and new types of DG without largely 'overcompensating' some DSOs that experience little or even no negative impact. The most sophisticated revenue driver seems to be a driver that accounts for all connected kW of DG capacity as well as for all kWh of DG electricity fed into the grid. Regarding the possible 'overcompensation' caused by regulatory improvements we noted that a minor 'overcompensation' might work in practice as an incentive to fully facilitate DG connection within their distribution network.

(7) Finally, it should be noted that the analysis in this paper is based on shallow network charging principles. Theoretically, DG operators could be given more efficient signals regarding location and timing of distributed electricity production, implying that network costs caused by DG operators will no longer be incurred by DSOs. However, successful implementation of time and location dependent network charges is not to be expected in the short to medium-run for technological, political and regulatory reasons.

The conclusions lead us to draw the following policy recommendations. Firstly, current regulation of DSOs should recognize the differential impact of increasing DG on DSO performance and should therefore investigate alternative regulation that can sufficiently take into account the drivers behind this impact. Secondly, as a specification of recommendation 1, we suggest to implement a regulatory formula where the impact of DG on both operating expenditures (with the main driver being electricity generated by DG units, impacting distribution losses) and capital expenditures (with the main drivers the amount of DG units connected to the distribution network) are included. Thirdly, in determining the specific values in the alternative regulatory formula we would recommend to aim for some 'overcompensation' of possible negative DG impact on DSOs in order to provide them with an explicit incentive to facilitate and accommodate new DG connections in their distribution networks. Fourthly, when considering the above recommendations on the neutralization and possible incentivisation of DSOs it should be kept in mind that the desirability of regulation aimed at these aspects is intertwined with the developments in the field of ancillary services provision by DSOs (as discussed in Jansen et al. (2007)). On the one hand, DSOs might need to be compensated for the negative impact following the penetration for DG. On the other hand, if DSOs benefit from the provision of ancillary services enabled by DG penetration, the DSO should compensate the DG operators. Netting both impacts implies that valuable economic signals are lost. Changes in regulation should therefore be targeted at either one, or both, described impacts. In the longrun, implementation of location and time dependent network charges could counter the negative impacts for DSOs described in this paper. However, considering the fact that DSOs in some countries are already experiencing the negative impact of large shares of DG in the network, solutions to this particular problem need to be found in adaptation of current regulation in the shortrun.

\section{Acknowledgements}

The analysis and results presented in this paper originate from research conducted for the DG-Grid project. This project was funded by the European Commission through the IEE programme (contract no. EIE/04/015/S07.38553). The authors thank the partners in the project for the cooperation and the valuable discussion on the analysis and results put forward in this paper.

\section{References}

Barth, R., Weber, C., Swider, D.J., 2008. Distribution of costs induced by the integration of RES-E power. Energy Policy 36 (8), 3107-3115.

Cao, D.M., Pudjianto, D., Grenard, S., Strbac, G., Martikainen, A., Kärkkäinen, S., Farin, J., 2006. Costs and Benefits of DG Connections to Grid System, Imperial College London Deliverable 8 of the DG-Grid project.

De Joode, J., Van der Welle, A.J., Jansen, J.C., 2007. Business models for DSOs under alternative regulatory regimes. ECN Report, ECN-E-07-038. Deliverable 10 of the DG-Grid project.

De Joode, J., Scheepers, M.J.J., Van der Welle, A.J., Jansen, J.C., 2007. Improving distribution network regulation for the enhancement of sustainable electricity supply. Proceedings of the 9th European IAEE Conference 2007, Florence, Italy, 10-13 June 2007.

Jansen, J.C., Van der Welle, A.J., De Joode, J., 2007. The evolving role of the DSO in efficiently accommodating distributed generation. ECN report, ECN-E-07-063. Deliverable 9 of the DG-Grid project. 
Jenkins, N., Allan, R.N., Crossley, P., Kirschen, D., Strbac, G., 2000. Embedded Generation. Institution of Electrical Engineers, London.

Knight, R.C., Montez, J.P., Knecht, F., Bouquet, T., 2005. Distribution generation connection charging within the European Union. Review of current practices, future options and European policy recommendations. Deliverable 2.1 (1) of the EU-Project ELEP-European Local Electricity Production, Cheshire.

McDonald, J., 2008. Adaptive intelligent power systems: Active distribution networks. Energy Policy 36, 4346-4351.

Mendez, V.H., Rivier, J., de la Fuente, J.I., Gomez, T., Arceluz, J., Marin, J., Madurga, A., 2006. Impact of distributed generation on distribution investment deferral. Electrical Power and Energy Systems 28, 244-252.

Pepermans, G., Driesen, J., Haeseldonckx, D., Belmans, R., D’haeseleer, W., 2005. Distributed generation: definition, benefits and issues. Energy Policy 33, 787-798.
Pudjianto, D., Cao, D.M., Grenard, S., Strbac, G., 2006. Method for Monetarisation of Cost and Benefits of DG Options. University of Manchester and Imperial College London, January 2006. Deliverable 7 of the DG-Grid project.

Skytte, K., Ropenus, S., 2007. Regulatory Review and Barriers for the Electricity Supply System for Distributed Generation in the EU-15. International Journal of Distributed Energy Resources 3, 243-257.

Strbac, G., 2008. Demand side management: Benefits and challenges. Energy Policy 36, 4419-4426.

Weber, C., Vogel, P., 2008. Assessing the benefits of a provision of system services by distributed generation. International Journal of Global Energy Issues 29, $162-180$.

Woodman, B., Baker, P., 2008. Regulatory frameworks for decentralised energy. Energy Policy 36, 4527-4531. 